\title{
BELIEF CONDENSATION FILTERING FOR RSSI-BASED STATE ESTIMATION IN INDOOR LOCALIZATION
}

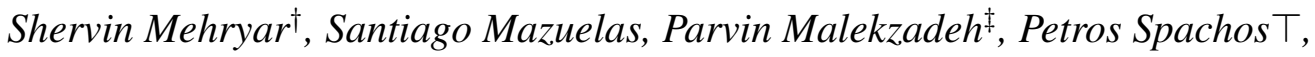 \\ Konstantinos N. Plataniotis ${ }^{\dagger}$, and Arash Mohammadi ${ }^{\ddagger}$ \\ $\dagger$ Department of Electrical and Computer Engineering, University of Toronto, Toronto, ON, Canada \\ $\ddagger$ Concordia Institute for Information System Engineering, Concordia University, Montreal, Canada \\ $\top$ School of Engineering, University of Guelph, Guelph, ON, Canada
}

\begin{abstract}
Recent advancements in signal processing and communication systems have resulted in evolution of an intriguing concept referred to as Internet of Things (IoT). By embracing the IoT evolution, there has been a surge of recent interest in localization/tracking within indoor environments based on Bluetooth Low Energy (BLE) technology. The basic motive behind BLE-enabled IoT applications is to provide advanced residential and enterprise solutions in an energy efficient and reliable fashion. Although recently different state estimation (SE) methodologies, ranging from Kalman filters, Particle filters, to multiple-modal solutions, have been utilized for BLEbased indoor localization, there is a need for ever more accurate and real-time algorithms. The main challenge here is that multipath fading and drastic fluctuations in the indoor environment result in complex non-linear, non-Gaussian estimation problems. The paper focuses on an alternative solution to the existing filtering techniques and introduce/discuss incorporation of the Belief Condensation Filter $(\mathrm{BCF})$ for localization via BLE-enabled beacons. The BCF is a member of the universal approximation family of densities with performance bound achieving accuracy and efficiency in sequential SE and Bayesian tracking. It is a resilient filter in harsh environments where nonlinearities and non-Gaussian noise profiles persist, as seen in such applications as Indoor Localization.
\end{abstract}

Index Terms - State Estimation, Belief Condensation, Bluetooth low energy (BLE), Indoor Localization, Internet of Things.

\section{INTRODUCTION}

The Internet of Things (Internet of Things (IoT)) [1-4] is a new emerging paradigm and is rapidly gaining ground in different applications of significant engineering importance including but not limited to smart home [5], medicare [6], smart industry [7], and smart public environments [8]. As is, patient serving devices in hospitals, energy saving appliances in households, and targeted advertising in consumer markets are a part of the benefits that arise from the IoT emergence. The main enabling factor of this promising paradigm is integration of identification, navigation, and localization technologies $[9,10]$ with smart hand-held devices equipped with sensing, processing, and communication capabilities. Bluetooth Low Energy (BLE), referred to as Bluetooth Smart [11, 12], is considered as the backbone technology for future indoor navigation [13] due to its high scan rate, very low power consumption, and better signal geometry. Another unique advantage of the BLE technology in IoT applications is in the growing number of BLE beacons. The ABI Research's report, "BLE Tags: The Location of Things" states that total BLE beacon shipments could exceed 400 million units in 2020 . With a standard and universally accepted architecture will follow a plethora of unknown applications that are yet to exceed expectations.

The archetypal system structure for smart applications, typically, consists of the following three main layers: (i) At the application level, user focused programs run on both mobile and anchored devices that turn data into applicable information; (ii) In the second layer, the data needed for user applications is distributed and shared among nodes, and; (iii) At the bottom layer, the communicated/shared data is collected, cut, and cleaned as to provide the foundation for the information and decision making monument. For an expanding system that heavily relies on estimations at every level, accuracy and minimized latency will become paramount with minimal tolerance for error. In different IoT applications that are mainly concerned with indoor micro localization-tracking, message passing and cooperative distributed estimation algorithms [14-17] are essential for proper integration of the aforementioned three layers, to take advantage of the richness of underlying data, and to achieve the high accuracy and low latency requirements of IoT applications. In this regard, to perform micro-localization based on BLE tags in IoT applications, the main-stream methodology is to use the Received Signal Strength Indicator (RSSI) [18]. The RSSIbased solutions, however, are prone to multipath fading and drastic fluctuations in the indoor environment [19]. A technical challenge in RSSI-based solutions is the presence of non-linearities at the lowlevel system. Such non-linearities have irrepressible precautions for estimation algorithms and can degrade performance if not properly dealt with. Another major hurdle for computing the exact states is the uncertainty and intermittence in the network. In most indoor environments, the exact form of the system noise is not known. Therefore, estimation algorithms must have built-in resilience to uncategorical noise models.

To deal with these issues within BLE-based indoor localization, multitude of advanced signal processing solutions are utilized. Kalman filters (KFs) [20,21] are used to smooth RSSI, Particle filters (PFs) [22] are incorporated to deal with non-linearities of the underlying model, Combination of linear (KFs) and non-linear techniques (PFs) are utilized, Gaussian sum filters [23], and multiple-model solutions [24] are used to deal with non-Gaussian and unknown noise characteristics. In this paper, we introduce/incorporate an alternative solution to the existing filtering techniques used recently for indoor localization/tracking via BLE-enabled beacons. The goal is to set forth a principled unifying framework to exploit contextual information. Given the scale, distributability, and precision necessary in IoT, the proposed framework aims to be compact, adaptive, and cascade minimal error through the system, and in so doing ac- 
commodate to the different needs of a content-rich network. In particular, the paper presents and incorporates the belief condensation filter $(\mathrm{BCF})[25,26]$, that provably performs well under non-linear, non-Gaussian conditions. The BCF provides a unified methodology for the design and analysis of different filtering techniques. The BCF can efficiently represent the complex distributions arising in RSSIbased filtering problems, and is obtained from an optimality criterion established based on a general framework for filtering techniques.

The reminder of the paper is organized as follows: Section 2 formulates the RSSI-based indoor localization problem. The BCF framework is introduced in Section 3. Experimental results and comparisons are provided in Section 4. Finally, Section 5 concludes the paper.

\section{PROBLEM FORMULATION}

For a system whose state evolves through time, the specific system dynamics can be described by a Hidden Markov Model (HMM). For example, in the case of indoor localization, which is the focus of this paper, the state vector $\boldsymbol{x}_{k}$ represents the coordinates of a moving agent in a building and the task of estimation is to predict the next state $\boldsymbol{x}_{k+1}$ and correct the outcome using RSSI measurements denoted by $\boldsymbol{y}_{k}$. For instance, in indoor localization/tracking problems using BLE-enabled beacons, nonlinearities within the RSSI can be captured by the following path loss model:

$$
\boldsymbol{y}_{k} \triangleq \mathrm{RSSI}=-10 n \log (d)+C,
$$

where $n$ represents path loss component, $d$ is the distance between the user and beacon, and $C$ is the average RSSI. The overall statespace model of the system is, therefore, given by:

$$
\begin{aligned}
\boldsymbol{x}_{k+1} & =g\left(\boldsymbol{x}_{k}\right)+\boldsymbol{q}_{k} \\
\boldsymbol{y}_{k} & =h\left(\boldsymbol{x}_{k}\right)+\boldsymbol{n}_{k},
\end{aligned}
$$

where $\boldsymbol{q}_{k}$ and $\boldsymbol{n}_{k}$ denote the dynamic model evolution and observation model uncertainty at time $k$, respectively. Eq. (2) is referred to as the Dynamic Model and Eq. (3) as the Observation Model. The HMM framework leads to the following two immediate assumptions:

1. The states $\boldsymbol{x}_{k}$ form a Markov chain, i.e., agent coordinates at a time $k$ only depend on those of the previous time step(s).

2. Observations $\boldsymbol{y}_{k}$ are independent given the states $\boldsymbol{x}_{k}$.

The joint distribution of state and observation variables, therefore, factors as follows:

$$
\begin{aligned}
f\left(\boldsymbol{x}_{1: k}, \boldsymbol{y}_{1: k}\right) & =\prod_{i=1}^{k} f\left(\boldsymbol{x}_{i} \mid \boldsymbol{x}_{i-1}\right) f\left(\boldsymbol{y}_{i} \mid \boldsymbol{x}_{i}\right) \\
& =f\left(\boldsymbol{x}_{1: k-1}, \boldsymbol{y}_{1: k-1}\right) f\left(\boldsymbol{x}_{k} \mid \boldsymbol{x}_{k-1}\right) f\left(\boldsymbol{y}_{k} \mid \boldsymbol{x}_{k}\right)(4)
\end{aligned}
$$

where $\boldsymbol{y}_{1: k}=\left\{\boldsymbol{y}_{1}, \ldots \boldsymbol{y}_{k}\right\}$. Computing the posterior density $f\left(\boldsymbol{x}_{k} \mid \boldsymbol{y}_{1: k}\right)$ as an inference problem, is therefore obtained by multiple applications of the Bayes rule:

$$
\begin{aligned}
f\left(\boldsymbol{x}_{k} \mid \boldsymbol{y}_{1: k}\right) & =\frac{f\left(\boldsymbol{y}_{k} \mid \boldsymbol{x}_{k}, \boldsymbol{y}_{1: k-1}\right) f\left(\boldsymbol{x}_{k} \mid \boldsymbol{y}_{1: k-1}\right)}{f\left(\boldsymbol{y}_{k} \mid \boldsymbol{y}_{1: k-1}\right)} \\
& =\frac{f\left(\boldsymbol{y}_{k} \mid \boldsymbol{x}_{k}\right) \int f\left(\boldsymbol{x}_{k-1} \mid \boldsymbol{y}_{1: k-1}\right) f\left(\boldsymbol{x}_{k} \mid \boldsymbol{x}_{k-1}\right) \mathrm{d} \boldsymbol{x}_{k-1}}{f\left(\boldsymbol{y}_{k} \mid \boldsymbol{y}_{1: k-1}\right)} .
\end{aligned}
$$

This computation referred to as the filtering process, can be decomposed into two steps:

$$
\begin{aligned}
f\left(\boldsymbol{x}_{k} \mid \boldsymbol{y}_{1: k-1}\right) & \propto \int f\left(\boldsymbol{x}_{k-1} \mid \boldsymbol{y}_{1: k-1}\right) f\left(\boldsymbol{x}_{k} \mid \boldsymbol{x}_{k-1}\right) \mathrm{d} \boldsymbol{x} \\
f\left(\boldsymbol{x}_{k} \mid \boldsymbol{y}_{1: k}\right) & \propto f\left(\boldsymbol{y}_{k} \mid \boldsymbol{x}_{k}\right) f\left(\boldsymbol{x}_{k} \mid \boldsymbol{y}_{1: k-1}\right) .
\end{aligned}
$$

This completes formulation of the non-linear, non-Gaussian estimation problem for performing indoor localization/tracking tasks via BLE-enabled IoT devices. Next, we present the BCF as an alternative filtering solution to the ones recently utilized in IoT applications via BLE beacons.

\section{BELIEF CONDENSATION FILTERING}

In this section, the details of the BCF filter are outlined, together with analysis that explores such advantages. The BCF is a filtering framework where the true posterior of the state vector is approximated by a mixture of probability density functions. It has been shown [26] that under certain optimality conditions, BCF can provide accuracies approaching the theoretical bounds and outperforming existing techniques, particularly for non-linear/non-Gaussian problems. One of the main advantages of the BCF filtering method is its treatment of the observation function as an inverse problem. While Kalmanlike filters tend to linearize the observation method (where needed) or approximate the posterior distribution with its Gaussian counterpart (e.g., in the case of Unscented Kalman filter), the BCF filter makes no assumptions as to what form $h(\cdot)$ must attain. This level of abstraction equips the BCF filter with interesting performance advantages while keeping the computational complexity low.

Consider the mixture family $\mathcal{F}_{\Xi^{m}}$ with an instance member $g(\boldsymbol{x} ; \xi)$ given by

$$
g(\boldsymbol{x} ; \xi)=\sum_{i=1}^{m} \alpha_{i} g_{i}\left(\boldsymbol{x} ; \theta_{i}\right),
$$

where $\alpha_{i} \in \mathbb{R}_{+}$, for $(1 \leq i \leq m) ; \sum_{i=1}^{m} \alpha_{i}=1$, and; $g_{i}\left(\boldsymbol{x} ; \theta_{i}\right)$, for $(1 \leq i \leq m)$, belongs to an exponential family $\mathcal{F}_{\Theta_{m}}$, i.e.,

$$
g_{i}(\boldsymbol{x} ; \xi)=q_{i}(\boldsymbol{x}) \exp \left\{\theta_{i}^{T} \mathbf{t}_{i}(\boldsymbol{x})-A_{i}\left(\theta_{\mathbf{i}}\right)\right\} .
$$

Here $\theta_{i} \in \Theta_{i}, \mathbf{t}_{i}(\boldsymbol{x})$, and $A_{i}\left(\theta_{i}\right)$ are the natural parameters, sufficient statistics, and $\log$-partition function of $\mathcal{F}_{\Theta_{i}}$. The parameter set for $g(\boldsymbol{x} ; \xi)$ consists of $\xi=\left(\alpha_{1}, \theta_{1}, \cdots, \alpha_{m}, \theta_{m}\right) \in \Xi^{m}$.

Let $f \in \mathcal{P}$ from the distribution family $\mathcal{P}$ denote the posterior distribution that we wish to approximate by $g(\boldsymbol{x} ; \xi) \in \mathcal{F}_{\Xi^{m}}$. For instance, in our particular analysis $f(\boldsymbol{x})=f\left(\boldsymbol{x}_{k} \mid \boldsymbol{y}_{1: k}\right)$. The Kullback-Leibler (KL) divergence $D_{K L}(\cdot)$ between the probability distributions $f(\boldsymbol{x})$ and $g(\boldsymbol{x} ; \xi)$ is defined as follows

$$
D_{K L}(f(\boldsymbol{x}), g(\boldsymbol{x} ; \xi))=\mathbb{E}_{f(\boldsymbol{x})}\left\{\log \frac{f(\boldsymbol{x})}{g(\boldsymbol{x} ; \xi)}\right\} .
$$

It can be shown that, under the following regularity conditions, $\mathrm{BCF}$ recursions (see Theorem 1), condense the probability distribution $f(\boldsymbol{x})$ into a mixture of exponential families:

(A1) The differential entropy of $f(\boldsymbol{x})$ is finite.

(A2) $\mathbb{E}_{f}\left\{\left|\log q_{i}(\boldsymbol{x})\right|\right\}$ is finite for each $i \in\{1,2, \cdots, m\}$

(A3) $\mathbb{E}_{f}\left\{\left|t_{i, j}(\boldsymbol{x})\right|\right\}$ is finite, where $t_{i, j}(\boldsymbol{x})$ is the $j$-th component of the sufficient statistic $\mathbf{t}_{i}(\boldsymbol{x})$, for $i \in\{1,2, \cdots, m\}$

(A4) The set $U_{f}=\left\{\xi \in \mathcal{F}_{\Xi^{m}}: D_{K L}\left(f, g_{\xi}\right)<\infty\right\}$ is open and non-empty 
Theorem 1 If a continuous probability distribution $f(\boldsymbol{x})$ satisfies the regularity conditions A1-A4, then the sequence $\left\{D_{K L}\left(f, g_{\xi^{[l]}}\right)\right\}_{l \in \mathbb{Z}_{+}}$ is monotonically decreasing, where the sequence $\left\{\xi^{[l]}\right\}_{l \in \mathbb{Z}_{+}} \subset \mathcal{F}_{\Xi^{m}}$ is recursively determined by

$$
\alpha_{i}^{[l+1]}=\alpha_{i}^{[l]} \mathbb{E}_{g_{i}\left(\boldsymbol{x} ; \theta_{i}^{[l]}\right)}\left\{\frac{f(\boldsymbol{x})}{g\left(\boldsymbol{x} ; \xi^{[l]}\right)}\right\}
$$

for $(1 \leq i \leq m)$, and $\theta_{i}^{[l+1]}$ satisfying

$$
\mathbb{E}_{g_{i}\left(\boldsymbol{x} ; \theta_{i}^{[l+1]}\right)}\left\{\mathbf{t}_{i}(\boldsymbol{x})\right\}=\frac{\mathbb{E}_{g_{i}\left(\boldsymbol{x} ; \theta_{i}^{[l]}\right)}\left\{\frac{f(x)}{g\left(x ; \xi^{[l]}\right)} \mathbf{t}_{i}(\boldsymbol{x})\right\}}{\mathbb{E}_{g_{i}\left(\boldsymbol{x} ; \theta_{i}^{[l]}\right)}\left\{\frac{f(x)}{g\left(x ; \xi^{[l]}\right)}\right\}} .
$$

for $(1 \leq i \leq m)$, and any initial parameter

$\xi^{[0]}=\left(\alpha_{1}^{[0]}, \theta_{1}^{[0]}, \alpha_{2}^{[0]}, \theta_{2}^{[0]}, \cdots, \alpha_{m}^{[0]}, \theta_{m}^{[0]}\right) \in U_{f}$.

In the case where the exponential families are Gaussian, $\theta_{i}^{[l+1]}$ in (12) can be obtained in a closed form as shown below.

Corollary 1 Let $\mathcal{F}_{\Xi^{m}}$ be the mixture family of $m$ Gaussian distributions, with each mixture component parameterized by $\theta_{i}=$ $\left\{\mu_{i}, \boldsymbol{\Sigma}_{i}\right\}$, i.e.,

$$
g_{i}\left(\boldsymbol{x} ; \theta_{i}\right) \sim \mathcal{N}\left(\mu_{i}, \boldsymbol{\Sigma}_{i}\right)
$$

If $f(\boldsymbol{x})$ is a continuous probability distribution satisfying the regularity conditions A1-A4, then the natural parameter $\theta_{i}^{[l+1]}$ at update step $(l+1)$ can be computed as

$$
\begin{aligned}
\mu_{i}^{[l+1]} & =\frac{\mathbb{E}_{g_{i}\left(\boldsymbol{x} ; \theta_{i}^{[l]}\right)}\left\{\frac{f(x)}{g\left(x ; \xi^{[l]}\right)} \boldsymbol{x}\right\}}{\mathbb{E}_{g_{i}\left(\boldsymbol{x} ; \theta_{i}^{[l]}\right)}\left\{\frac{f(x)}{g\left(x ; \xi^{[l]}\right)}\right\}} \\
\boldsymbol{\Sigma}_{i}^{[l+1]} & =\frac{\mathbb{E}_{g_{i}\left(\boldsymbol{x} ; \theta_{i}^{[l]}\right)}\left\{\frac{f(x)}{g\left(x ; \xi^{[l]}\right)} \boldsymbol{x} \boldsymbol{x}^{T}\right\}}{\mathbb{E}_{g_{i}\left(\boldsymbol{x} ; \theta_{i}^{[l]}\right)}\left\{\frac{f(x)}{g\left(x ; \xi^{[l]}\right)}\right\}}-\mu_{i}^{[l+1]}\left(\mu_{i}^{[l+1]}\right)^{T} .
\end{aligned}
$$

Eqs. (13) and (14) provide a recursive method for calculating and updating the state variables in each step. The main complexity in this computation comes from carrying out the computation for the expectation integrals. The fact that these expectations are taken with respect to a member of an exponential family (namely a Gaussian distribution) can be exploited, for which efficient quadrature rules exist [27]. In this case, these integrals can be efficiently computed with polynomial time in $m$ the number of components; $q$ the number of quadrature points, and; $d$ the dimension of the state vector.

Example 1 (A Non-linear Model) Consider an observation model where we try to estimate parameter $x \in \mathbb{R}$ given $N$ i.i.d. noisy observations $y_{i} \in \mathbb{R}$. In particular, imagine the relation between the observation $y$ and state parameter $x$ to be expressed by the nonlinear function $h(x)=x^{2} \sin (x)$, which gives rise to the following observation model:

$$
y_{i}=x^{2} \sin (x)+n_{i}, \quad i=1,2, \cdots N
$$

where $n_{i}$ are independent, additive noise terms distributed according to a normal Gaussian distribution with variance $\sigma_{n}^{2}=0.1$. To characterize the performance of the BCF algorithm, a Monte Carlo simulation is run and the mean squared error (MSE) over 100 trials is computed. In each trial, we generate $N=5$ noisy observations from the true value of $x=2.2$ and make point estimation using

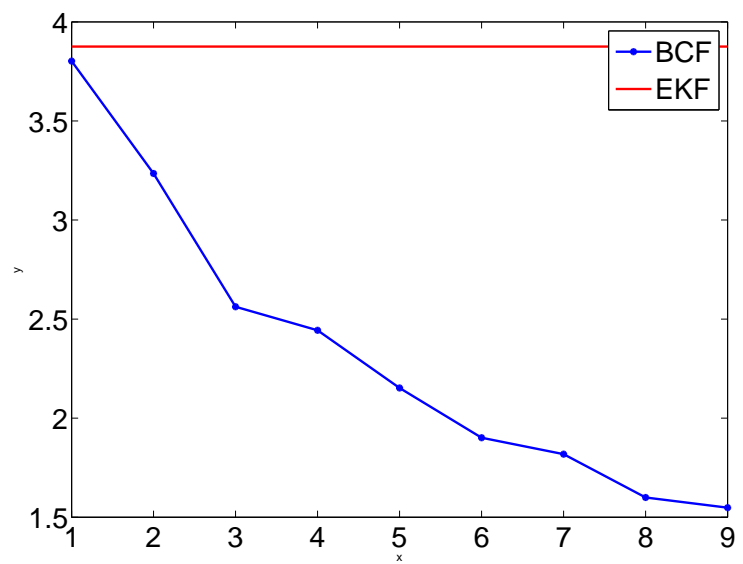

Fig. 1. MSE plot for a non-linear model using BCF and EKF.

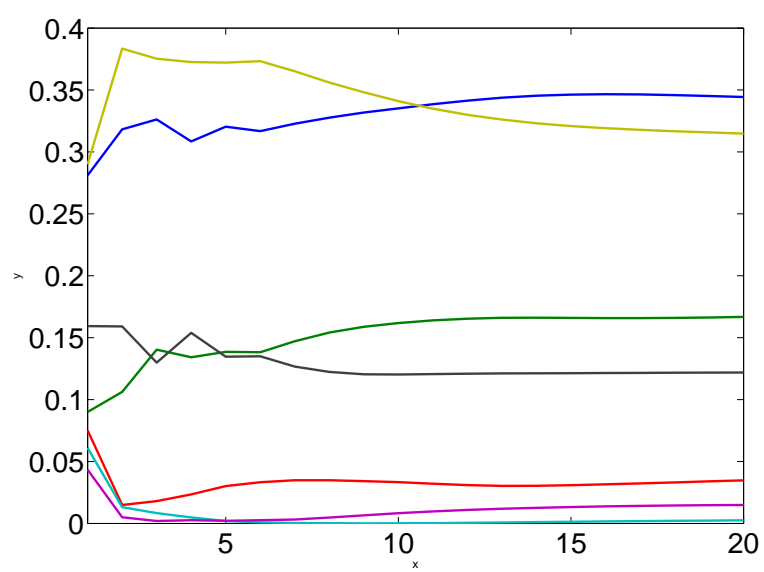

Fig. 2. Convergence plots of $\alpha_{i}$ 's in the BCF algorithm.

both BCF and extended Kalman filter (EKF) methods. We use the mean of the posterior as the point estimate. The results are shown in Figure 1. From the figure, it can be seen that in the presence of nonlinearity in the model, the BCF outperforms the Kalman filter due to linearization assumptions such methods make. It can also be seen that, with increasing $m$, the accuracy in estimation for BCF also improves. Figure 2 shows the convergence behavior of the coefficients $\alpha_{i}$ which in general settle after $l=5$ to $l=10$ steps.

\section{NUMERICAL EXPERIMENTATION AND ANALYSIS}

In this section, we apply the BCF methodology to the problem of state estimation (SE) in Indoor Localization. We adopt the test setup in [18] and use the RSSI measurements to perform location estimation for a stationary device. The measurements are collected indoors by three BLE beacons located at coordinates $(0,0),(d, 0)$, and $(d, d)$, with $d=5 m$, and the device rests at coordinates $\left(\frac{2 d}{3}, \frac{d}{3}\right)$. The parameters for the environment model of Equation (1) are given as $n=2.511$ and $C=75.54$. Since the device is not in motion, i.e. $g(\cdot)=\mathbb{1}(\cdot)$ of Equation (2), the problem boils down to correcting the position using the relation in Equation (7). We generate $K=500$ initial positions $\boldsymbol{x}_{k}$, and take 90 independent measurements $\boldsymbol{y}_{k}$ to perform estimation $\hat{\boldsymbol{x}}_{k}$ of the state vector in relation 


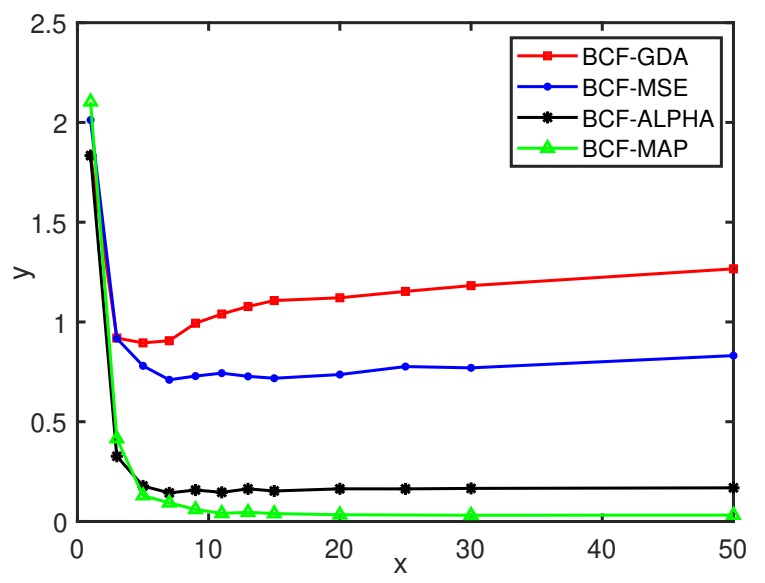

Fig. 3. MSE computation for the BCF as a function of the number of mixture components $m$. Point estimation is performed by: (i) BCFGDA: posterior mean constrained to $\boldsymbol{\Sigma}_{i}=\boldsymbol{\Sigma}_{j}, \forall i, j$; (ii) BCF-MSE: posterior mean; (iii) BCF-ALPHA: posterior mode constrained to $\boldsymbol{\Sigma}_{i}=\boldsymbol{\Sigma}_{j}, \forall i, j$; (iv) BCF-MAP: posterior mode.

\begin{tabular}{||c|c|c|c||}
\hline \multicolumn{2}{|c|}{ BCF-MSE } & \multicolumn{2}{c|}{ PF } \\
\hline$m$ & MSE $[\mathrm{m}]$ & $n$ & MSE $[\mathrm{m}]$ \\
\hline 5 & 0.70 & 10 & 1.3 \\
\hline 7 & 0.49 & 50 & 0.68 \\
\hline 9 & 0.44 & 100 & 0.47 \\
\hline 11 & 0.38 & 300 & 0.49 \\
\hline 13 & 0.32 & 500 & 0.46 \\
\hline 15 & 0.32 & 1000 & 0.50 \\
\hline
\end{tabular}

Table 1. MSE values in meters, for BCF as a function of component number $m$ vs PF as a function of particle number $n$.

$\boldsymbol{y}_{k}=h\left(\boldsymbol{x}_{k}\right)+\boldsymbol{n}_{k}$, each trial. The noise here is assumed to be i.i.d. Gaussian and the point estimation is done using four different methods. Each method is evaluated with an aggregate MSE:

$$
\mathrm{MSE}=\frac{1}{K} \sum_{k=1}^{K}\left\|\boldsymbol{x}_{k}-\hat{\boldsymbol{x}}_{k}\right\|^{2}
$$

where here the number of trials $K=500$. This is done for different settings of BCF with $m=1,3, \cdots, 50$ components. The measurement data $\boldsymbol{y}_{k}$ are provided in the public database of [18]. The question here is, under equal dynamic and noise models, what accuracy (MSE) and efficiency (computation time) does the BCF achieve?

Figure 3 shows the MSE as a function of mixture components $m$ for $\mathrm{BCF}$, for four different point estimation methods. In the case of BCF-GDA, the covariance matrices of the BCF components are set equal $\left(\boldsymbol{\Sigma}_{i}=\boldsymbol{\Sigma}_{j}, \forall i, j \in\{1 \cdots m\}\right)$. This behaves as Gaussian Discriminant Analysis, a linear boundary classifier, and with increasing components $m$ tends to deteriorate. The BCF-MSE estimate corresponds to taking the mean of the posterior $f\left(\boldsymbol{x}_{k} \mid \boldsymbol{y}_{1: k}\right)$, which achieves localization accuracies within $70 \mathrm{~cm}$ at $m=9$. The BCF-ALPHA favors the data by optimizing coefficients $\alpha_{i}$, i.e. fixed $\left(\mu_{i}, \boldsymbol{\Sigma}_{i}\right)$. Its location error lies under $15 \mathrm{~cm}$. The final curve, MSEMAP estimates location by computing the mode of the posterior distribution. The MAP estimate is most resilient to sparsity in data.

Table 3 shows the MSE for BCF against the PF filter in one instantiation of the process. At starting from $m=7$ the same levels

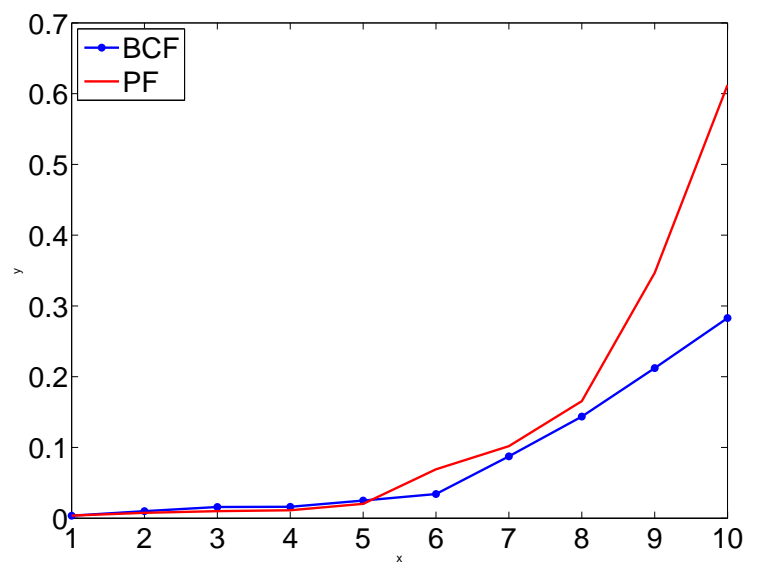

Fig. 4. Complexity analysis in terms of computation time as a function of state dimension $(d)$ for the BCF and PF algorithms at $r=2$.

of accuracy as PF with high number of particles $n=1000$ is obtainable. In the current literature, it is claimed that in the limit PF indeed can achieve the theoretical bounds of estimation accuracy. On the other hand, it is known that PF suffers from the curse of dimensionality as it utilizes Monte Carlo methods to approximate multidimensional integrals [28-30]. We pursue and compare here the computation time of the $\mathrm{BCF}$ and $\mathrm{PF}$ through numerical experimentation. We adopt a methodology presented in [31], whereby a dimension free metric for error is introduced. Adopting the notation of [31], let $r$ denote the Mean Dimension-Free Error defined by:

$$
r=\frac{\mathbb{E}\left\{(\boldsymbol{x}-\hat{\boldsymbol{x}})^{*} J(\boldsymbol{x}-\hat{\boldsymbol{x}})\right\}}{d}
$$

where $\hat{\boldsymbol{x}}$ is the estimate of $\boldsymbol{x}$ from the BCF or PF, $d$ is the state dimension, $J$ is the inverse of the estimation error covariance matrix, $\boldsymbol{x}$ is the state vector to be estimated, and $(\cdot)^{*}$ denotes the transpose of $(\cdot)$. First the number of mixture components $m$ or particles $n$ is selected that obtain a fixed value of $r$. The complexity of each filter is then defined as the time it takes the algorithms to reach $r$. From Figure 4, at the same accuracy, we see the benefit in choosing BCF carrying far less components over PF's computational burden.

\section{CONCLUSION}

Due to the nature of the sensory networks of the IoT, it is logical to view its evolutionary dynamics in a probabilistic framework. On par with the wide spread use of Bayesian networks, the estimation process must be condensed, resilient, and transferable. Although recently different SE approaches within Bayesian formulation have been used for BLE-based indoor localization, the achievable overall accuracies are still limited. This is mainly due to the multipath fading and drastic fluctuations in the indoor environment resulting in complex non-linear, non-Gaussian estimation problems. To tackle these problems, different linear and non-linear estimators such as Kalman filters, Particle filters, Gaussian sum filters, and multiplemodel techniques have been utilized for BLE-based localization. In this paper, we focus on an alternative solution to the existing filtering techniques and introduce/discuss incorporation of the BCF for indoor tracking via BLE-enabled beacons. The paper shows that the $\mathrm{BCF}$ is a suitable candidate for the ubiquitous networks of the future. 


\section{REFERENCES}

[1] P. Spachos, I. Papapanagiotou and K.N. Plataniotis, "Microlocation for Smart Buildings in the Era of the Internet of Things: A Survey of Technologies, Techniques, and Approaches," IEEE Signal Processing Magazine, vol. 35, no. 5, pp. 140-152, Sept. 2018.

[2] C. Xu, L. Yang and P. Zhang, "Practical Backscatter Communication Systems for Battery-Free Internet of Things: A Tutorial and Survey of Recent Research," IEEE Signal Processing Magazine, vol. 35, no. 5, pp. 16-27, Sept. 2018.

[3] M. Z. Win, F. Meyer, Z. Liu, W. Dai, S. Bartoletti and A. Conti, "Efficient Multi-sensor Localization for the Internet of Things: Exploring a New Class of Scalable Localization Algorithms," IEEE Signal Processing Magazine, vol. 35, no. 5, pp. 153-167, Sept. 2018.

[4] J. Prieto, A. Amira, J. Bajo, S. Mazuelas, and F. De la Prieta, "Iot Approaches for Distributed Computing," Wirel. Commun. Mob. Comput., vol. 2018, pp. 40-, Mar. 2018.

[5] F. Amirjavid, P. Spachos, K.N. Plataniotis, "3-D Object Localization in Smart Homes: A Distributed Sensor and Video Mining Approach," IEEE Systems Journal, vol. 12, no. 2, pp. 1307-1316, June 2018.

[6] Z. Iqbal, D. Luo, P. Henry, S. Kazemifar, T. Rozario, Y. Yan, K. Westover, W. Lu, D. Nguyen, T. Long, J. Wang, H. Choy, and S. Jiang, "Accurate Real Time Localization Tracking in a Clinical Environment using Bluetooth Low Energy and Deep Learning," arXiv/1711.08149, 2017.

[7] G. Kalantar, A. Mohammadi, S.N. Sadrieh, "Analyzing the Effect of Bluetooth Low Energy (BLE) with Randomized MAC Addresses in IoT Applications," IEEE Inter. Conf. Internet of Things, 2018.

[8] S. Alletto et al., "An Indoor Location-Aware System for an IoT-Based Smart Museum," IEEE Internet of Things Journal, vol. 3, no. 2, pp. 244-253, April 2016.

[9] A. Kushki, K.N. Plataniotis, A.N. Venetsanopoulos, "WLAN Positioning Systems: Principles and Applications in Location based Services," Cambridge University Press, 2013.

[10] A. Kushki, K.N. Plataniotis, A.N. Venetsanopoulos, "Kernelbased Positioning in Wireless Local Area Networks," IEEE Trans. on Mobile Computing, vol. 6, no. 6, pp. 689-705, June 2007.

[11] P. Spachos, and A. Mackey, "Energy Efficiency and Accuracy of Solar Powered BLE Beacons," Computer Communications, vol. 119, pp. 94-100, 2018.

[12] Y. Gu and F. Ren, "Energy-Efficient Indoor Localization of Smart Hand-Held Devices Using Bluetooth," IEEE Access vol. 3, no. , pp. 1450-1461, 2015.

[13] P. Davidson; R. Piche, "A Survey of Selected Indoor Positioning Methods for Smartphones," in IEEE Communications Surveys \& Tutorials, vol. 19, no. 2, pp. 1347-1370, 2017.

[14] A. Mohammadi, S. Davar and K. N. Plataniotis, “TernaryEvent-Based State Estimation With Joint Point, Quantized, and Set-Valued Measurements," IEEE Signal Processing Letters, vol. 25, no. 5, pp. 665-669, 2018.

[15] A. Amini, A. Asif, A. Mohammadi, “"CEASE: A Collaborative Event-Triggered Average-Consensus Sampled-Data Framework With Performance Guarantees for Multi-Agent Systems," IEEE Trans. Signal Processing, vol. 66, no. 23, pp. 6096-6109, Dec. 2018.
[16] A. Mohammadi and K. N. Plataniotis, "Distributed Widely Linear Multiple-Model Adaptive Estimation," IEEE Transactions on Signal and Information Processing over Networks, vol. 1, no. 3, pp. 164-179, Sept. 2015.

[17] A. Mohammadi and A. Asif, "Distributed Consensus+Innovation Particle Filtering for Bearing/ Range Tracking With Communication Constraints," IEEE Trans. Signal Processing, vol. 63, no. 3, pp. 620-635, 2015.

[18] S. Sadowski and P. Spachos, "RSSI-Based Indoor Localization With the Internet of Things," IEEE Access, vol. 6, pp. 3014930161, 2018.

[19] S. Aditya, A. F. Molisch and H. M. Behairy, "A Survey on the Impact of Multipath on Wideband Time-of-Arrival Based Localization," Proceedings of the IEEE, vol. 106, no. 7, pp. 1183-1203, July 2018.

[20] V.C. Paterna, A.C. Auge, J.P. Aspas, and M.A. Perez Bullones, "A Bluetooth Low Energy Indoor Positioning System with Channel Diversity, Weighted Trilateration and Kalman Filtering," Sensors, vol. 17, no. 12, 2017.

[21] J. Robesaat, P. Zhang, M. Abdelaal, O. Theel, "An Improved BLE Indoor Localization with Kalman-Based Fusion: An Experimental Study," Sensors, vol. 17, 2017.

[22] F. Zafari, I. Papapanagiotou, M. Devetsikiotis, and T.J. Hacker, "An Ibeacon based Proximity and Indoor Localization System," CoRR, vol. abs/1703.07876, 2017.

[23] A. Mohammadi, K.N. Plataniotis: "Complex-Valued Gaussian Sum Filter for Nonlinear Filtering of Non-Gaussian/NonCircular Noise," IEEE Signal Process. Lett., vol. 22, no. 4, pp. 440-444, 2015.

[24] F.S. Danis and A.T. Cemgil, "Model-based Localization and Tracking using Bluetooth Low-Energy Beacons," Sensors, vol. 17, no. 11, 2017.

[25] H. Wymeersch, J. Lien, and M.A. Win, "Cooperative Localization in Wireless Networks," Proceedings of the IEEE, vol. 97, no. 2, pp. 427-450, Feb 2009.

[26] S. Mazuelas, Y. Shen and M. Z. Win, "Belief Condensation Filtering," IEEE Transactions on Signal Processing, vol. 61, no. 18, pp. 4403-4415, Sept.15, 2013.

[27] L. Arasaratnam, S. Haykin, and T.R. Hurd, "Cubature kalman filtering for continuous-discrete systems: Theory and simulations," vol. 58, no. 10, pp. 4977-4993, Oct 2010.

[28] S. Arulampalam and B. Ristic, "Comparison of the particle filter with range-parameterized and modified polar ekfs for angleonly tracking," in AeroSense 2000. International Society for Optics and Photonics, 2000, pp. 288-299.

[29] Stanton Musick, John L. Greenewald, Keith Kastella, and Chris Kreucher, "Comparison of particle method and finite difference nonlinear filters for low snr target tracking," in Proceedings of International Conference on Fusion, Aug 2001.

[30] Alfonso Farina, Branko Ristic, and Dario Benvenuti, "Tracking a ballistic target: comparison of several nonlinear filters," vol. 38, no. 3, pp. 854-867, Jul 2002.

[31] Fred Daum and Jim Huang, "Curse of dimensionality and particle filters," in Aerospace Conference, 2003. Proceedings. 2003 IEEE, March 2003, vol. 4, pp. 1979-1993. 